08

\title{
Зависимость скорости роста и структуры III-V нитевидных нанокристаллов от площади сбора адатомов на поверхности подложки
}

\author{
() В.Г. Дубровский \\ Санкт-Петербургский государственный университет, Санкт-Петербург, Россия \\ E-mail: dubrovskii@mail.ioffe.ru
}

Поступило в Редакцию 5 февраля 2021г

В окончательной редакции 5 февраля 2021г.

Принято к публикации 10 февраля 2021 г.

\begin{abstract}
Проведено теоретическое исследование зависимости скорости роста и структуры нитевидных нанокристаллов полупроводниковых соединений III-V от площади диффузионного сбора адатомов на поверхности подложки. Получено выражение для максимальной скорости роста нитевидных нанокристаллов. Проанализированы причины ее уменьшения в различных технологиях. Показано, что скорость роста пропорциональна площади сбора и обратно пропорциональна квадрату радиуса нанокристалла. Продемонстрировано, что автокаталитические нитевидные нанокристаллы GaAs расширяются при больших и сужаются при малых значениях площади сбора и при этом в обоих случаях обладают кубической структурой. Вюрцитная фаза формируется в области промежуточных значений площади сбора.
\end{abstract}

Ключевые слова: нитевидный нанокристалл, полупроводниковые соединения III-V, механизм роста пар-жидкость-кристалл, скорость роста, площадь сбора адатомов.

DOI: 10.21883/PJTF.2021.09.50906.18727

Нитевидные нанокристаллы (ННК) полупроводниковых соединений III-V представляют большой интерес как для исследований ростовых процессов и фундаментальных физических свойств, так и для приложений в нанофотонике, в особенности совмещенной с кремниевой электронной платформой [1]. Чрезвычайно эффективная релаксация упругих напряжений на развитой боковой поверхности позволяет выращивать III-V ННК на поверхностях кремния, а также комбинировать материалы с большим рассогласованием решеток в гетероструктурах на основе ННК без формирования дислокаций несоответствия [2]. Следует отметить, что бездислокационный рост в сильно рассогласованных системах, таких как InAs/Si (параметр рассогласования $\varepsilon_{0}=11.6 \%$ ), трудно осуществимый в планарных технологиях [3], в геометрии ННК требует лишь их достаточно малого диаметра (менее $25 \mathrm{~nm}$ ). III-V ННК обычно синтезируются по механизму пар-жидкость-кристалл (ПЖК) [4], где в качестве металлических катализаторов роста используются либо $\mathrm{Au}[5,6]$, либо элемент III группы (Ga для НHК GaAs) при автокаталитическом ПЖК-росте [7]. Последний позволяет не только полностью устранить возможное проникновение $\mathrm{Au}$ в ННК, но и использовать стандартные технологии подготовки регулярных массивов отверстий в оксидном слое $\mathrm{SiO}_{x}$ на поверхности $\mathrm{Si}(111)$, в которые осаждаются капли $\mathrm{Ga}[8,9]$. При этом экспериментальные данные указывают на нетривиальную зависимость скорости роста, морфологии и даже кристаллической фазы HНK GaAs от шага регулярной решетки массива отверстий $P[8,9]$. Поэтому целью настоящей работы является теорети- ческое исследование зависимости указанных характеристик III-V ННК от площади сбора адатомов группы III (Ga) на поверхности подложки.

Определим сначала максимально возможную скорость вертикального роста ННК. Для этого необходимы: 1) полное отсутствие десорбции атомов группы III с поверхности и из капель катализатора роста; 2) отсутствие радиального роста ННК, т. е. их постоянный во времени и не зависящий от длины ННК радиус $R$. Для идеального ансамбля ННК цилиндрической формы, имеющих одинаковый радиус и длину $L$, закон сохранения полного числа атомов группы III, осаждаемых на поверхность из газового потока $v_{3}[\mathrm{~nm} / \mathrm{s}]$, дает

$$
v_{3} t=\pi R^{2} L N=\frac{\pi R^{2} L}{P^{2}},
$$

где $N=1 / P^{2}-$ поверхностная плотность ННК. Из (1) следует

$$
L=v_{3} t \frac{P^{2}}{\pi R^{2}}, \quad \frac{d L}{d t}=v_{3} \frac{P^{2}}{\pi R^{2}} .
$$

Как уже указывалось, в случае литографически процессированных подложек $P$ есть расстояние между отверстиями, а $R$ есть радиус основания капли. При спонтанном формировании капель, например при термическом отжиге слоя $\mathrm{Au}[5,6], P$ есть среднее расстояние между ННК, зависящее от толщины слоя $\mathrm{Au}$, температуры и времени отжига. Такой процесс приводит к естественной дисперсии по размерам $R$ со средним значением $\langle R\rangle$; обе эти величины также зависят от указанных выше параметров процесса отжига. Для 
неидеального ансамбля ННК можно записать

$$
v_{3} t=\int d R f(R) \pi R^{2} L(R, t)
$$

где $f(R)$ - функция распределения ННК по радиусу, удовлетворяющая условию нормировки $\int d R f(R)=N=1 / P^{2}$. Единственным решением данного уравнения будет длина $L$, определяемая формулой (2).

Согласно (2), длина ННК пропорциональна эффективной толщине осажденного материала $v_{3} t$ (в условиях, обогащенных по группе V) и коэффициенту ускорения роста $P^{2} /\left(\pi R^{2}\right)$, который показывает, во сколько раз скорость роста ННК больше скорости роста двумерного слоя в тех же условиях. Естественно, что ускорение происходит за счет селективности роста исключительно под каплями катализатора. Например, при $P=300 \mathrm{~nm}$ и $R=30 \mathrm{~nm}$ коэффициент ускорения равен 32, а соответствующая скорость вертикального роста ННК при типичной для молекулярно-пучковой эпитаксии (МПЭ) скорости осаждения $\mathrm{Ga} v_{3}=0.3 \mathrm{~nm} / \mathrm{s}$ равна примерно $10 \mathrm{~nm} / \mathrm{s}$. Увеличение $P$ до $1000 \mathrm{~nm}$ при тех же прочих значениях приводит к коэффициенту ускорения 1110 и скорости роста ННК, равной $330 \mathrm{~nm} / \mathrm{s}$, что едва ли достижимо. Перечислим основные причины замедления скорости роста ННК по сравнению с максимальным теоретическим значением: 1) двумерный рост паразитного слоя на подложке $[5,6,10] ; 2)$ десорбция атомов группы III с поверхности твердого тела или из жидкой капли $[5,11]$; 3) радиальный рост ННК [6-9]; 4) лимитированный потоком элемента группы V автокаталитический рост [7-9,12,13], что обычно также приводит к радиальному росту. Первая и вторая причины могут быть устранены за счет использования селективных оксидных масок и понижения температуры поверхности соответственно, хотя десорбция $\mathrm{Ga}$ или In из капли требует отдельного исследования. Отметим также, что выражения (2) представляют собой простейший вид ранее исследованных моделей роста III-V НHК с Аu-катализаторами $[6,14,15]$, в которых зависимость $L \propto 1 / R^{2}$ возникала за счет доминирующей диффузии адатомов группы III с поверхности подложки.

Скорость роста (или длина) III-V ННК, обратно пропорциональная радиусу, также неоднократно наблюдалась экспериментально $[6,16]$. В частности, на рис. 1 показана зависимость длины Аu-каталитических ННК InP от их диаметра из работы [16], которая очень хорошо описывается формулой (2) при $P=62 \mathrm{~nm}$. Отметим, однако, что такое расстояние между ННК соответствовало бы их поверхностной плотности $2.6 \cdot 10^{10} \mathrm{~cm}^{-2}$, а измеренная на основании данных [12] плотность ННК на порядок меньше. Следовательно, далеко не все атомы осажденного In входят в состав ННК. В данном случае замедление роста ННК связано прежде всего с ростом паразитного слоя InP, поскольку данные ННК выращивались методом МПЭ на подложках $\operatorname{InP}(111) B$

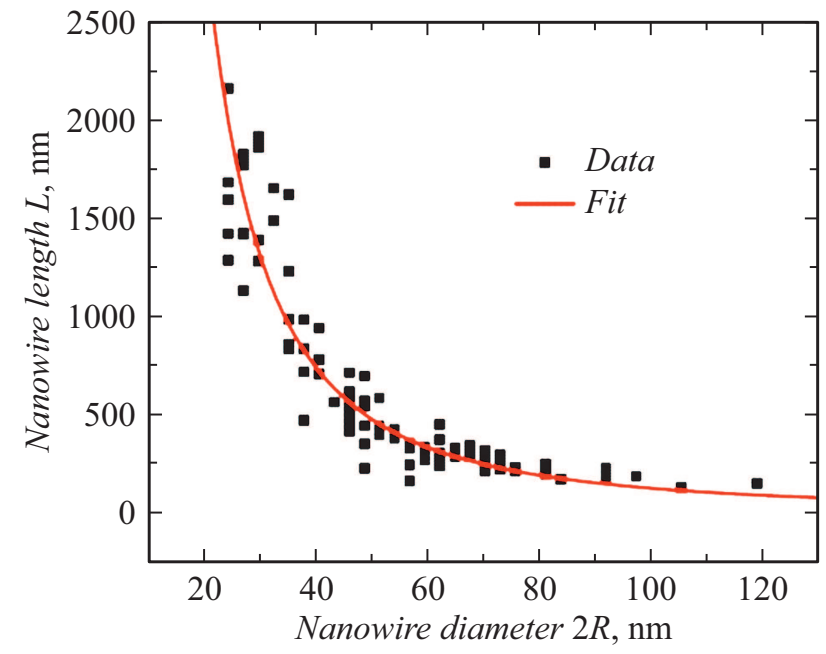

Рис. 1. Зависимость длины от диаметра ННК InP, выращенных методом МПЭ с Аu-катализаторами на подложках $\operatorname{InP}(111) B$ при температуре $420^{\circ} \mathrm{C}$ [16] (символы), и ее аппроксимация, полученная из уравнения (2) при $v_{3} t=250 \mathrm{~nm}$ (экспериментальное значение) и $P=62 \mathrm{~nm}$ (линия).

из капель $\mathrm{Au}$, полученных термическим отжигом слоя $\mathrm{Au}$ толщиной $1 \mathrm{~nm}$.

Рассмотрим теперь автокаталитический процесс ПЖК, при котором ННК GaAs не только растут вертикально, но и изменяют свой диаметр, который может увеличиваться или уменьшаться в зависимости от соотношения потоков $\mathrm{As} / \mathrm{Ga}[8,9,17]$. Предполагая, как и ранее, отсутствие десорбции $\mathrm{Ga}$, для цилиндрического ННК с $L \gg R$ имеем

$$
\frac{d}{d t}\left(\pi R^{2} L\right)=v_{3} P^{2},
$$

т. е. изменение объема ННК равно потоку $\mathrm{Ga}$, приходящемуся на данный ННК. С другой стороны, скорость вертикального роста автокаталитического ННК пропорциональна атомарному потоку As $v_{5}$ :

$$
\frac{d L}{d t}=\chi_{5} v_{5}
$$

В случае МПЭ геометрический коэффициент $\chi_{5}\left(\alpha_{5}, \beta\right)$ зависит от угла падения потока As $\alpha_{5}$ и контактного угла капли $\beta$ согласно [18].

В соответствии с результатами работ $[19,20]$ капля $\mathrm{Ga}$ на вершине HНК GaAs имеет два стабильных угла: $\beta_{\min } \cong 100^{\circ}$ и $\beta_{\max } \cong 127^{\circ}$, между которыми ННК растут без изменения радиуса. В области $100<\beta<125^{\circ}$ ННК формируются в гексагональной вюрцитной (WZ) кристаллической фазе, а вне ее - в кубической фазе цинковой обманки (ZB), характерной для объемного GaAs [19]. Узкая область $125<\beta<127^{\circ}$ соответствует HНК GaAs в фазе ZB без бокового роста. При $\beta=127^{\circ}$ ННК расширяются, а при $\beta=100^{\circ}$ сужаются, т. е. происходит изменение их радиуса при $\beta=$ const [20] (более 


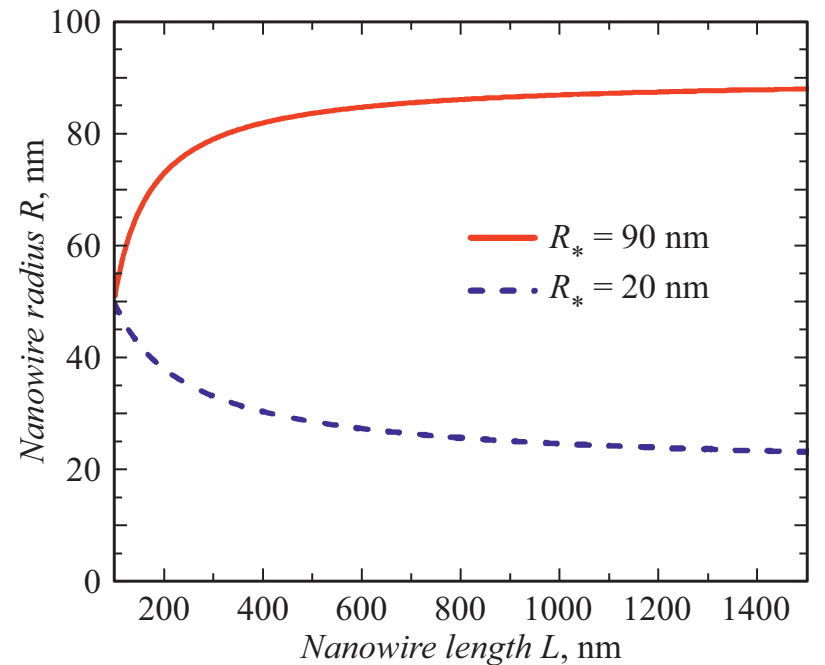

Рис. 2. Характерные зависимости радиуса автокаталитических III-V ННК от их длины, полученные из формулы (6) для ННК с начальным радиусом $50 \mathrm{~nm}$ при $R_{*}=90 \mathrm{~nm}$ (расширяющиеся ННК при большой площади диффузионного сбора адатомов) и $R_{*}=20 \mathrm{~nm}$ (сужающиеся ННК при малой площади диффузионного сбора адатомов).

сложная геометрия конусовидных ННК с расширением или сужением от основания к вершине здесь не рассматривается). При $\beta=\mathrm{const,} \mathrm{используя} \mathrm{(4)} \mathrm{и} \mathrm{(5),} \mathrm{получаем}$

$$
R^{2}=R_{*}^{2}-\frac{\left(R_{*}^{2}-R_{0}^{2}\right) L_{0}}{L},
$$

где $L_{0}$ - длина ННК, имеющего радиус $R_{0}$ по окончании короткой стадии инкубации. Очевидно, что радиус ННК стремится к стационарному значению $R_{*}$, которое зависит от контактного угла:

$$
\begin{aligned}
& R_{\text {max }}^{2}=R_{*}^{2}\left(\beta_{\min }\right)=\frac{v_{3}}{\chi_{5}\left(\beta_{\min }\right) v_{5}} \frac{P^{2}}{\pi}, \\
& R_{\text {min }}^{2}=R_{*}^{2}\left(\beta_{\max }\right)=\frac{v_{3}}{\chi_{5}\left(\beta_{\max }\right) v_{5}} \frac{P^{2}}{\pi} .
\end{aligned}
$$

При углах падения потока As, меньших $40^{\circ}$, можно использовать выражение $\chi_{5}\left(\beta_{\max }\right)=1 / \sin ^{2} \beta_{\max }$ для расширяющихся ННК. При $\beta_{\min } \cong 100^{\circ}$ выражение $\chi_{5} \cong\left(1+\cos \alpha_{5}\right) / 2$ является хорошим приближением для сужающихся ННК [18].

Таким образом, при $R_{0}<R_{\min }$ должно наблюдаться увеличение радиуса ННК от $R_{0}$ до $R_{\min }$ при контактном угле капли $\beta_{\max }$, а при $R_{0}>R_{\max }-$ уменьшение радиуса ННК от $R_{0}$ до $R_{\min }$ при контактном угле капли $\beta_{\min }$. Типичные морфологии показаны на рис. 2 . Кристаллическая фаза расширяющихся или сужающихся HНK GaAs должна быть ZB. В случае промежуточных значений начального радиуса $R_{\min }<R_{0}<R_{\max }$ ННК должны расти только вертикально в кристаллической фазе WZ. Согласно (7), стационарные радиусы ННК пропорциональны $P$. Следовательно, при заданном диапазоне размеров капель (определяющих значения $R_{0}$ ) большие значения $P$ (ННК малой поверхностной плотности) будут соответствовать расширяющимся ННК, а малые (ННК большой поверхностной плотности) сужающимся. Кристаллическая фаза в обоих случаях будет ZB, при этом расширяющиеся ННК будут содержать меньше дефектов $[19,20]$. Промежуточные значения $P$ будут соответствовать исключительно вертикальному росту при промежуточных значениях контактных углов $\beta_{\min }<\beta<\beta_{\max }$, преимущественно (при $\beta<125^{\circ}$ ) в фазе WZ. Можно предположить, что аналогичные качественные закономерности окажутся справедливыми и для других III-V ННК. Это обстоятельство открывает новую возможность управления морфологией и кристаллической фазой III-V ННК за счет изменения расстояния между каплями катализатора роста.

В заключение отметим, что в работе предложена простая модель роста III-V ННК в зависимости от характерного расстояния $P$, с которого происходит сбор адатомов группы III на поверхности подложки. Показано, что максимальная скорость роста ННК прямо пропорциональна $P^{2}$ и обратно пропорциональна квадрату радиуса ННК. Показано, что при автокаталитическом росте ННК GaAs изменение $P$ позволяет получать различные морфологии (сужающиеся, расширяющиеся и постоянного радиуса) в разных кристаллических фазах. Проведенное исследование позволяет заключить, что расстояние между ННК, легко изменяемое при росте в регулярных массивах литографически подготовленных отверстий, является важнейшим контрольным параметром, определяющим свойства III-V ННК.

\section{Финансирование работы}

Работа выполнена при поддержке гранта Санкт-Петербургского государственного университета № 61520973.

\section{Конфликт интересов}

Автор заявляет, что у него нет конфликта интересов.

\section{Список литературы}

[1] A. Zhang, G. Zheng, C.M. Lieber, Nanowires: building blocks for nanoscience and nanotechnology(Springer, 2016).

[2] F. Glas, Phys. Rev. B, 74, 121302(R) (2006). DOI: 10.1103/PhysRevB.74.121302

[3] G.E. Cirlin, V.G. Dubrovskii, V.N. Petrov, N.K. Polyakov, N.P. Korneeva, V.N. Demidov, A.O. Golubok, S.A. Masalov, D.V. Kurochkin, O.M. Gorbenko, N.I. Komyak, V.M. Ustinov, A.Yu. Egorov, A.R. Kovsh, M.V. Maximov, A.F. Tsatusul'nikov, B.V. Volovik, A.E. Zhukov, P.S. Kop'ev, Zh.I. Alferov, N.N. Ledentsov, M. Grundmann, D. Bimberg, Semicond. Sci. Technol., 13, 1262 (1998). DOI: $10.1088 / 0268-1242 / 13 / 11 / 005$

[4] R.S. Wagner, W.C. Ellis, Appl. Phys. Lett., 4, 89 (1964). DOI: $10.1063 / 1.1753975$ 
[5] V.G. Dubrovskii, I.P. Soshnikov, G.E. Cirlin, A.A. Tonkikh, Yu.B. Samsonenko, N.V. Sibirev, V.M. Ustinov, Phys. Status Solidi B, 241, R30 (2004). DOI: 10.1002/pssb.200409042

[6] V.G. Dubrovskii, N.V. Sibirev, R.A. Suris, G.E. Cirlin, J.C. Harmand, V.M. Ustinov, Surf. Sci., 601, 4395 (2007). DOI: $10.1016 /$ j.susc.2007.04.122

[7] C. Colombo, D. Spirkoska, M. Frimmer, G. Abstreiter, A. Fontcuberta i Morral, Phys. Rev. B, 77, 155326 (2008). DOI: 10.1103/PhysRevB.77.155326

[8] V.G. Dubrovskii, T. Xu, A. Díaz Álvarez, G. Larrieu, S.R. Plissard, P. Caroff, F. Glas, B. Grandidier, Nano Lett., 15, 5580 (2015). DOI: 10.1021/acs.nanolett.5b02226

[9] S. Plissard, G. Larrieu, X. Wallart, P. Caroff, Nanotechnology, 22, 275602 (2011). DOI: 10.1088/0957-4484/22/27/275602

[10] J.C. Harmand, F. Glas, G. Patriarche, Phys. Rev. B, 81, 235436 (2010). DOI: 10.1103/PhysRevB.81.235436

[11] V.G. Dubrovskii, J. Grecenkov, Cryst. Growth Design, 15, 340 (2015). DOI: $10.1021 / \mathrm{cg} 5014208$

[12] F. Glas, M.R. Ramdani, G. Patriarche, J.C. Harmand, Phys. Rev. B, 88, 195304 (2013). DOI: 10.1103/PhysRevB.88.195304

[13] M.R. Ramdani, J.C. Harmand, F. Glas, G. Patriarche, L. Travers, Cryst. Growth Design, 13, 91 (2013). DOI: $10.1021 / \mathrm{cg} 301167 \mathrm{~g}$

[14] B.M. Borg, J. Johansson, K. Storm, K. Deppert, J. Cryst. Growth, 366, 15 (2013). DOI: 10.1016/j.jcrysgro.2012.12.142

[15] J. Johansson, M.H. Magnusson, J. Cryst. Growth, 525, 125192 (2019). DOI: 10.1016/j.jcrysgro.2019.125192

[16] R.J. Yee, S.J. Gibson, V.G. Dubrovskii, R.R. LaPierre, Appl. Phys. Lett., 101, 263106 (2012). DOI: 10.1063/1.4773206

[17] J. Tersoff, Nano Lett., 15, 6609 (2015). DOI: 10.1021/acs.nanolett.5b02386

[18] F. Glas, Phys. Status Solidi B, 247, 254 (2010). DOI: $10.1002 / p s s b .200945456$

[19] F. Panciera, Z. Baraissov, G. Patriarche, V.G. Dubrovskii, F. Glas, L. Travers, U. Mirsaidov, J.C. Harmand, Nano Lett., 20, 1669 (2020). DOI: 10.1021/acs.nanolett.9b04808

[20] W. Kim, V.G. Dubrovskii, J. Vukajlovic-Plestina, G. Tütüncüoglu, L. Francaviglia, L. Güniat, H. Potts, M. Friedl, J.-B. Leran, A. Fontcuberta i Morral, Nano Lett., 18, 49 (2018). DOI: 10.1021/acs.nanolett.7b03126 